\title{
Long-term outcomes of very low birth weight infants with spontaneous intestinal perforation: A retrospective case-matched cohort study ${ }^{\text {is }}$
}

\author{
Isabelle Adant ${ }^{a}$, Marc Miserez ${ }^{b}$, Gunnar Naulaers ${ }^{c, d}$, Kate Carkeek ${ }^{c}$, Els Ortibus ${ }^{d}$, Raf Aerts ${ }^{e}$, Maissa Rayyan ${ }^{c, *}$ \\ a Department of Pediatrics, University Hospitals Leuven, Belgium Herestraat 49, B-3000 Leuven, Belgium \\ b Department of Abdominal Surgery, University Hospitals Leuven, Belgium Herestraat 49, B-3000 Leuven, Belgium \\ c Department of Neonatology, University Hospitals Leuven, Belgium Herestraat 49, B-3000 Leuven, Belgium \\ d Department of Development and Regeneration, KU Leuven, Belgium Herestraat 49, B-3000, Leuven, Belgium \\ e Health Impact Assessment, Sciensano (Belgian Institute of Health) Juliette Wytsmanstraat 14, B-1050, Brussels, Belgium
}

\section{A R T I C L E I N F O}

Article history:

Received 11 September 2018

Received in revised form 10 April 2019

Accepted 13 April 2019

Available online $\mathrm{xxxx}$

\section{Key words:}

Spontaneous intestinal perforation

Neurodevelopmental outcome

Quality of life

Preterm

Very low birthweight infant

\begin{abstract}
A B S T R A C T
Background: Spontaneous intestinal perforation (SIP) is an intestinal complication that occurs in very ill preterms. We investigated whether SIP survivors have worse neurodevelopmental and gastrointestinal outcomes and a poorer quality of life than controls.

Methods: A retrospective case-matched cohort study was performed involving infants treated for SIP in a NICU between August 1994 and April 2014. Controls and SIP patients were matched to gestational age, gender, and birth period. Medical records were reviewed. Telephone surveys were conducted to evaluate the medical condition, quality of life (PedsQL ${ }^{\mathrm{TM}} 4.0$ ), neuropsychiatric and gastrointestinal outcome. McNemar's and Wilcoxon tests were performed, and generalized linear models were computed.

Results: Forty-nine SIP patients were included. The percentages of children with multiple disabilities (40\% vs. 17\%, $\mathrm{OR}=3.3$ ) and requiring physiotherapy ( $86 \%$ vs. $60 \%, \mathrm{OR}=4.77$ ) were higher in the SIP group than in the control group. Intraventricular hemorrhage (IVH) led to a worse neurodevelopmental outcome regardless of SIP (OR $=$ 8.79 for disability), and female gender was a protective factor against disability ( $O R=0.06)$. Reported quality of life and gastrointestinal comorbidities did not differ between the two groups.

Conclusion: SIP survivors tend to be at risk of multiple disabilities. IVH and female gender influence the neurodevelopmental outcome regardless of SIP.

Levels of evidence: Level III: case-control study.
\end{abstract}

(c) 2019 Elsevier Inc. All rights reserved.
Spontaneous intestinal perforation (SIP) is a life-threatening condition that affects $2 \%-3 \%$ of very low birth weight infants (VLBWIs, birth weight $<1500 \mathrm{~g}$ ). This complication is defined as a (mostly) single spontaneous perforation at the antimesenteric border of the bowel, most often at the terminal ileum [1]. Although the exact etiology of this condition is unknown, several possible risk factors have been identified such as male gender, severe chorioamnionitis, and the use of indomethacin and steroids [2-4]. Neonates affected by SIP typically present with abdominal distension and discoloration, and systemic involvement can also be observed [3,5]. Diagnosis is confirmed by detection of pneumoperitoneum on abdominal radiography [3,5]. SIP must be differentiated from necrotizing enterocolitis (NEC). Patient history and radiological/histological characteristics can help in this regard [3,5-8]. Therapeutic options include

\footnotetext{
it Funding: This research did not receive any specific grant from funding agencies in the public, commercial, or not-for-profit sectors.

* Corresponding author at: University Hospitals Leuven, Neonatal Intensive Care Unit, Herestraat 49, B-3000 Leuven, Belgium. Tel.: + 3216343211.

E-mail address: maissa.rayyan@uzleuven.be (M. Rayyan).
}

laparotomy and primary peritoneal drainage [6,9]. Mortality is high (20\%-40\%). Rates of neonatal morbidity, such as a longer hospital stay, requirement for mechanical ventilation, and delay of enteral nutrition, are high among survivors $[4,7,8,10]$. In addition, the prevalence of neurodevelopmental impairment among VLBWIs is high [11].

Over the past decades, it has become clear that inflammatory disease, in addition to hypoxic-ischemic insults, is an important cause of perinatal brain damage $[12,13]$. Thus, patients with SIP can be expected to have neurological consequences. However, there are scarce data regarding the long-term neurological and gastrointestinal outcomes and quality of life of SIP patients $[7,8,10,14]$. To the best of our knowledge, only two groups have analyzed the neurodevelopmental outcome of SIP patients. They reported that the prevalence of neurodevelopmental impairment ranges from $26 \%$ to $62.8 \%$. [4,15]. We were unable to find any studies investigating the gastro-intestinal outcome of SIP patients.

We conducted the first case-matched cohort study comparing the long-term developmental and gastrointestinal outcomes and quality of life of preterm infants with SIP and preterm matched controls without SIP. We investigated the hypothesis that SIP patients have worse 
developmental and gastrointestinal outcomes and a poorer quality of life than matched preterm controls.

\section{Methods}

\subsection{Patients and study design}

This retrospective case-control cohort study was approved by the local ethical committee of the University Hospitals Leuven (Belgium). Patients with SIP who were treated at the tertiary neonatal intensive care unit (NICU) of the University Hospitals Leuven between August 1, 1994, and March 31, 2014, were included. The clinical files of preterm infants born at $\leq 32$ weeks of gestation were reviewed [16]. SIP was diagnosed based on clinical, radiological, and histological findings (when available). Patients with suspected NEC, mechanical gastrointestinal obstruction, and congenital malformations were excluded from the study. Controls were matched for gender and gestational age (equal, plus or minus 1 week) and were born as close as possible to the birth date of their matched patients. In multiple pregnancies where one infant developed SIP, a sibling of the same gender was used as the control when possible. Exclusion criteria for controls were neonatal death, NEC requiring surgery, and associated congenital malformations. Each SIP patient was matched with one control patient. To compare illness severity during the neonatal period, the neonatal characteristics (e.g., CRIB II score [17], bronchopulmonary dysplasia, and retinopathy of prematurity requiring treatment) of patients and controls were recorded [16].

\subsection{Data collection}

All available clinical neonatal, neurological, mental health, and gastrointestinal follow-up data were reviewed. VLBWIs were followed up closely by a multidisciplinary team at corrected ages of 7-11 and 18-22 months. Bayley Scales of Infant Development II (BSID II) were scored by trained psychologists and physiotherapists to evaluate cognitive and motor development [18]. Further clinical evaluations were conducted depending on the child's needs. Results of BSID II scoring and intelligence tests (IQ) were noted whenever they were performed.

To evaluate long-term well-being, a telephone survey was conducted between January 2015 and September 2017 by two (para)medically trained staff (see list 1, Appendix A). Parents were asked questions about the general medical condition (biometrics, medical history, and current medication use) of their child, the use of rehabilitation services (such as speech therapy, physiotherapy, psychotherapy, and homebased care) by their child, and the type of school education of their child. In Belgium, the regular educational system (composed of preschool, primary school, and three types of secondary school) is distinguished from the specialized educational system. The latter is adapted to special needs children with the following disabilities: developmental delay, motor problems, hearing impairment, visual impairment, mental health problems, learning disturbances, or a combination thereof.

To evaluate neurodevelopmental impairment, functional disabilities were classified into the following four categories: severe, moderate, mild, and no disability. Diagnosis was performed by an experienced neuropediatrician based on the criteria used in the EPIPAGE studies [19]. Severe disability was defined as nonambulatory cerebral palsy $(\mathrm{CP})$, severe bilateral hearing loss, severe visual loss that persisted even with visual correction ( $<3 / 10$ bilaterally), or severe cognitive disability (IQ of less than 55). Moderate disability was classified as CP requiring the use of walking aids, moderate cognitive disability (IQ of 55-69), refractory epilepsy and severe learning disorders requiring an adapted educational system. Mild disability was classified as ambulatory $\mathrm{CP}$, mild cognitive disability (IQ of 70-85), unilateral visual loss $(<3 / 10$ unilaterally), epilepsy controlled by medication, and mild conduct problems (compatible with the regular educational system). Children without disabilities were classified into the "no disability" category. Children with disabilities in more than one functional field (e.g., motor problems and visual impairment) were classified as having multiple disabilities. When parents reported mental health problems, medical files were reviewed in order to obtain a confirmed diagnosis. Mental health disorders were classified into 4 categories: attention problems, internalizing and externalizing problems, conduct disorders and autism spectrum disorders. For the analysis of mental health issues, we only included patients with confirmed diagnosis of mental health disorder.

Weight, height, and weight for height were converted into standard deviations using the most recent Flemish reference growth charts [20]. Use of the following six types of medication was recorded: laxatives, antidiarrheal drugs, anticonvulsants, growth hormones, asthma drugs, and muscle relaxants. Use of laxatives and antidiarrheal drugs was indicative of impaired bowel function, use of anticonvulsants was indicative of severe brain damage, and use of psychotropic drugs was indicative of mental health problems. Growth hormones can be prescribed to preterm infants who are small for their gestational age and whose growth does not catch up.

Gastrointestinal problems were screened by conducting a short survey based on the PedsQL ${ }^{\mathrm{TM}}$ Gastrointestinal Symptoms Scales [21,22]. A validated Dutch translation of these scales is unavailable.

Quality of life was quantified using the validated PedsQL ${ }^{\mathrm{TM}}$ Quality of Life Inventory, version 4.0, in Dutch $[23,24]$. Parents were questioned about their child.

\subsection{Data analysis}

Data of SIP patients and matched controls were statistically compared using SPSS software (SPSS Statistics for Windows, Version 25.0; SPSS Inc., Chicago). Categorical data were analyzed using McNemar's test, and noncategorical data were analyzed using the paired Wilcoxon test. Given the matched nature of this study, only SIP patients and their matched controls for whom data were collected were included in statistical analyses. $\mathrm{p}<0.05$ was considered significant.

Subsequently, multivariate analyses were performed, meaning to control for potential confounding by the matching variables [25]. We identified 5 potential risk factors for outcome namely the matching criteria (gender, gestational age, birth weight), SIP and IVH. Models were computed for the main outcome parameters (BSID II, disability and multiple disabilities, regular educational system, PedsQL ${ }^{\mathrm{TM}}$ and gastrointestinal questionnaire) and for the outcome parameters that were significantly different in the univariate analysis. On the one hand, generalized linear models (GLMs) with a gamma distribution and the log-link function were used to estimate the predictors of health-related quality of life (PedsQL ${ }^{\mathrm{TM}}$ ), BSID II and growth parameters [26]. On the other, binary logistic model was used to estimate the predictors of all binomial data (e.g., disability, physiotherapy). Intraventricular hemorrhage (IVH) (grade 3 and 4), intestinal perforation and gender were set as fixed factors in the models, while birthweight and gestational age were set as covariates. The main effects and an intercept were modeled, and odds ratios and their 95\% Wald confidence limits were calculated for all factors and covariates. Akaike's information criterion (AIC) was calculated to assess the multivariate model quality.

\section{Results}

\subsection{Study subjects (Fig. 1)}

Between August 1, 1994, and March 31, 2014, 3131 neonates born at $\leq 32$ weeks of gestation were admitted. Sixty-four (2.04\%) patients were diagnosed with SIP. Two patients were excluded owing to associated congenital malformations requiring surgery. Thirteen (21.0\%) patients died during the neonatal period. No patients with SIP died after discharge. Forty-nine SIP patients were finally included in the study, and these patients were matched with 49 controls according to the aforementioned criteria. 


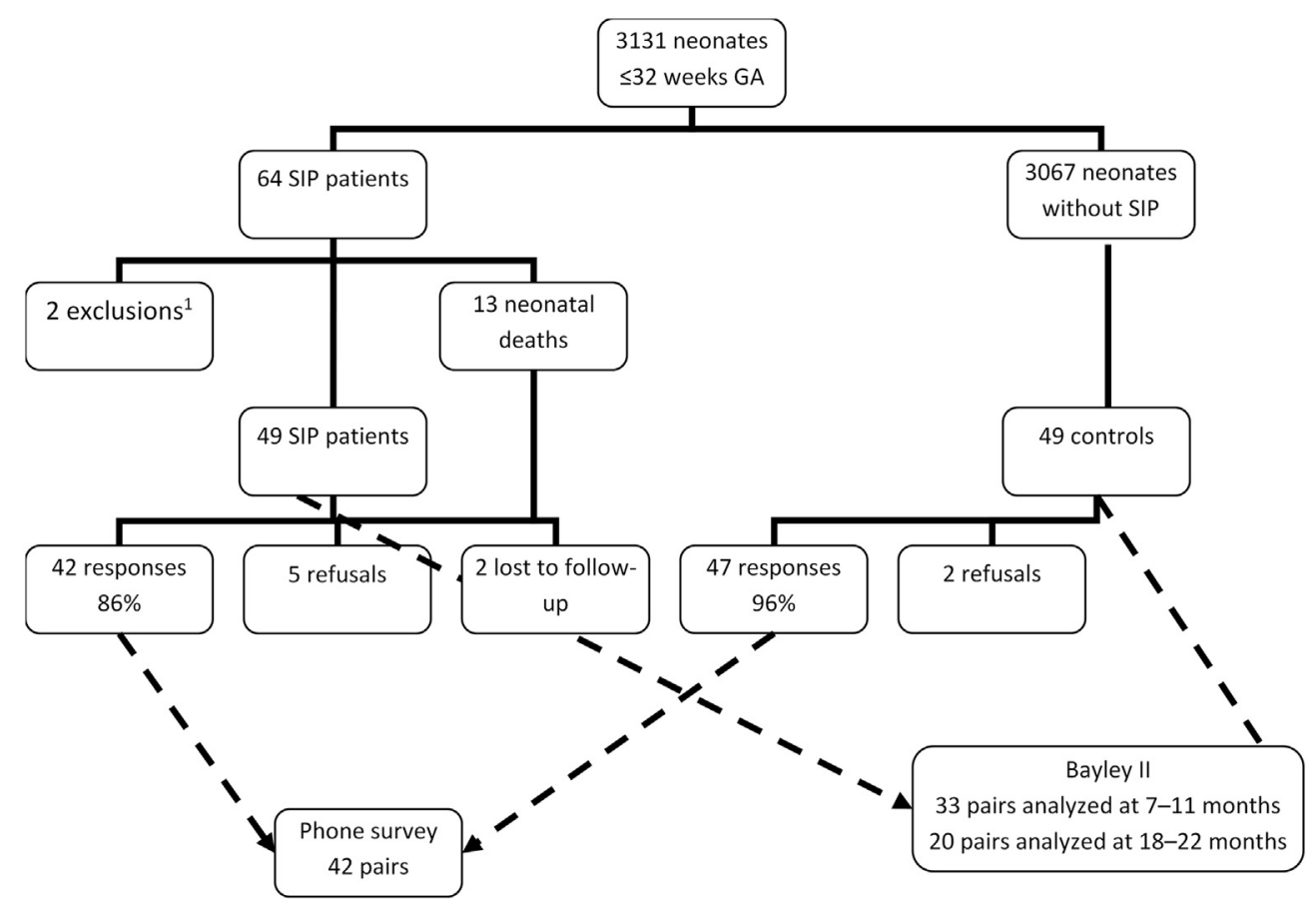

Fig. 1. Study design. ${ }^{1}$ Due to multiple congenital malformations.

The parents of 42 (86\%) SIP patients completed the telephone questionnaire; the parents of five patients refused to participate, and two patients were lost to follow-up (Fig. 1). The parents of 47 (96\%) controls completed the telephone survey; the parents of two controls refused to participate. Median age at evaluation was 13 years in both groups (range, 3-20 years). Data of $42(85.7 \%)$ SIP patients and matched controls were analyzed (Fig. 1). The characteristics of the SIP patients and their matched controls are described in Table 1.

For Bayley neurodevelopmental indices, 33 (67\%) pairs were evaluated at 7-11 months (median age: 9 months) and 20 (40.8\%) pairs were reevaluated at 18-22 months (median age: 19 months). In both groups, 24 (48.9\%) children underwent IQ testing when 4-10 years old. Among these, data of only 14 pairs of SIP patients and matched controls could be analyzed.

\subsection{Neurodevelopmental outcome (Tables 2, 3)}

Results from the univariate analysis are summarized in Table 2 (unless multivariate analysis was stated). Table 3 summarizes the Odds Ratios obtained from the multivariate analyses of the data.

Table 1

Demographic and clinical characteristics of SIP patients and controls.

\begin{tabular}{lll}
\hline Variable & $\begin{array}{l}\text { SIP patients } \\
\mathrm{N}=42\end{array}$ & $\begin{array}{l}\text { Controls } \\
\mathrm{N}=42\end{array}$ \\
\hline Male gender, $\mathrm{n}(\%)^{\text {Birth weight (g) }}{ }^{\mathrm{a}}$ & $33(78.5)$ & $33(78.5)$ \\
Gestational age (weeks.days) $^{\mathrm{a}}$ & $929.0 \pm 97.9$ & $975.2 \pm 86.9$ \\
Ventilation (days) $^{\mathrm{a}}{ }^{*}$ & $26.6 \pm 4.6$ & $26.9 \pm 3.9$ \\
Days to full enteral nutrition $^{\mathrm{a}}{ }^{*}$ & $\mathbf{2 6 . 4} \pm \mathbf{6 . 9}$ & $\mathbf{9 . 7} \pm \mathbf{4 . 2}$ \\
Length of stay (days) $^{\mathrm{a}}{ }^{*}$ & $\mathbf{5 4 . 8} \pm \mathbf{7 . 7}$ & $\mathbf{3 8 . 4} \pm \mathbf{7 . 9}$ \\
Bronchopulmonary dysplasia (\%) $^{\mathrm{b}}$ & $\mathbf{1 1 4 . 3} \pm \mathbf{1 2 . 1}$ & $\mathbf{7 6 . 4} \pm \mathbf{1 0 . 2}$ \\
${\text { Retinopathy requiring treatment }(\%)^{\mathrm{b}}}_{\text {CRIB II score }^{\mathrm{a}}}$ & $21 \pm 6.2(50)$ & $17 \pm 6.2(40.5)$ \\
\hline
\end{tabular}

Significant data marked in bold.

a Mean \pm SD.

b $\mathrm{n} \pm \mathrm{SD}$.

c Ventilation was defined as an infant being on conventional or high-frequency ventilation.

* Significance level: $\mathrm{p}<0.05$.
During their stay at the NICU, 13 (31\%) SIP patients developed an IVH of grade 3 or higher. The prevalence of hydrocephalus at discharge was higher in the SIP group than in the control group (21.4\% vs. 7.1\%; $\mathrm{OR}=8.139 ; \mathrm{p}=0.01 ; \mathrm{GLM})$. Hydrocephalus appeared to be related to severe IVH in this SIP group (OR =65.195; $p<0,0001$; GLM). Eight SIP patients required a ventriculoperitoneal shunt (19.0\%). Periventricular leukomalacia was diagnosed based on transfontanellar ultrasonography in both SIP patients and controls (11.9\% vs. 14.2\%; NS).

The BSID II PDI score was worse in the SIP group than in the control group at a corrected median age of 19 months $(\mathrm{OR}=0.847 ; \mathrm{p}=0.04$; GLM). The prevalence of CP was similar in the SIP and control groups (16.7\% vs $14.3 \%)$.

Many preterm infants receive rehabilitation therapy later in life, and this was the case for both SIP patients and controls. The percentage of children who received physiotherapy was significantly higher in the SIP group than in the control group (85.7\% vs 59.5\%; OR = 4.8; $\mathrm{p}=$ 0.01 ; GLM). The main reasons for starting physiotherapy were developmental delay (38.1\%) and hypertonia (23.8\%).

The percentage of disabled children was higher in the SIP group compared to the control group (59.5\% vs. $40.5 \%$; OR $=2.04$; NS; GLM). Seventeen SIP patients had multiple disabilities, in comparison with only seven controls ( $40.5 \%$ vs. $16.7 \%$; OR $=3.3 ; \mathrm{p}=0.04$; GLM). Fig. 2 shows determinants of (multiple) disability calculated by multivariate analysis. The percentage of children who attended special needs schools was almost 2-fold higher in the SIP group than in the control group, although this difference was not significant ( $45.2 \%$ vs. $25.2 \%$; $\mathrm{OR}=1.77 ; \mathrm{p}=0.29 ; \mathrm{GLM})$. Multifactorial testing revealed that this difference was because the number of patients with IVH was higher in the SIP group than in the control group ( $O R=8.73 ; p=0,009 ; \mathrm{GLM})$. Female gender was a protective factor against disability $(\mathrm{OR}=0.06$; $\mathrm{p}=0.001 ; \mathrm{GLM})$, multiple disabilities (OR $=0.196 ; \mathrm{p}=0.04 ; \mathrm{GLM})$, and the requirement for special needs education $(\mathrm{OR}=0.172 ; \mathrm{p}=$ 0.03; GLM) in our cohort, regardless of intestinal perforation or IVH. Appendix B contains information about the quality of multivariate model fitting.

Parents of 15 SIP survivors and 8 controls reported mental health problems. Review of the available medical files provided accurate diagnoses in 15 SIP patients and 7 controls (35.7\% vs. $16.7 \%$; OR $=2.68$; $\mathrm{p}=$ 
Table 2

Comparative analysis of SIP patients and controls.

\begin{tabular}{|c|c|c|c|c|c|}
\hline$\underline{\text { Variable }}$ & & $\frac{\text { SIP patients }}{\text { Number }(\%)}$ & $\frac{\text { Controls }}{\text { Number }}(\%)$ & $\underline{\text { P-value }}$ & Odds ratio \\
\hline \multicolumn{6}{|l|}{ Neurologic outcome } \\
\hline Normal brain ultrasound at admission & & $26(61.9 \%)$ & $25(59.5 \%)$ & 1 & 1.11 \\
\hline Intraventricular hemorrhage (grade $\geq 3$ ) & & $13(31.0 \%)$ & $6(14.3 \%)$ & 0.09 & 2.69 \\
\hline Normal brain ultrasound at discharge ${ }^{a}$ & & $16(38.1 \%)$ & $28(66.7 \%)$ & 0.06 & 0.353 \\
\hline Hydrocephalus at discharge ${ }^{* a}$ & & $11(21.4 \%)$ & $3(7.1 \%)$ & 0.01 & 8.139 \\
\hline Periventricular white matter disease & & $5(11.9 \%)$ & $6(14.2 \%)$ & 1 & 0.81 \\
\hline \multirow{4}{*}{ BSID II } & MDI at 7-9 months & 74.8 & 77.4 & 0.64 & \\
\hline & PDI at 7-9 months & 94.3 & 92.5 & 0.72 & \\
\hline & MDI at $18-20$ months $^{\mathrm{a}}$ & 72.8 & 84.2 & 0.40 & 0.941 \\
\hline & PDI at 18-20 months*a & 91.4 & 89.8 & 0.04 & 0.847 \\
\hline \multirow[t]{8}{*}{ Rehabilitation services } & Speech therapy & $32(76.2 \%)$ & $29(69.0 \%)$ & 0.55 & 1.43 \\
\hline & Physiotherapy*a & $36(85.7 \%)$ & $25(59.5 \%)$ & 0.01 & 4.77 \\
\hline & Developmental delay & $16(38.1 \%)$ & $8(19.0 \%)$ & 0.06 & 2.62 \\
\hline & Hypertonia & $10(23.8 \%)$ & $11(26.2 \%)$ & 1 & 0.88 \\
\hline & Psychotherapy & $10(23.8 \%)$ & $12(28.6 \%)$ & 0.80 & 0.78 \\
\hline & Home based care & $19(45.2 \%)$ & $15(35.7 \%)$ & 0.45 & 1.49 \\
\hline & Wearing glasses ${ }^{* a}$ & $22(52.4 \%)$ & $12(28.6 \%)$ & 0.02 & 3.084 \\
\hline & Wearing hearing aids & $3(7.1 \%)$ & $2(4.8 \%)$ & 1 & 1.54 \\
\hline \multirow[t]{2}{*}{ Follow-up } & Tertiary referral center & $18(42.9 \%)$ & $12(28.6 \%)$ & 0.18 & 1.88 \\
\hline & Psychiatric & $6(14.3 \%)$ & $2(4.8 \%)$ & 0.22 & 3.33 \\
\hline Education & Regular educational system ${ }^{a}$ & $21(54.8 \%)$ & $31(74.8 \%)$ & 0.29 & 1.77 \\
\hline \multirow[t]{5}{*}{ Medication use } & Psychotropic drugs & $8(19.0 \%)$ & $4(9.5 \%)$ & 0.34 & 2.24 \\
\hline & Anticonvulsant drugs & $5(11.9 \%)$ & $1(2.4 \%)$ & 0.22 & 5.54 \\
\hline & Asthma medication & $3(7.1 \%)$ & $2(4.8 \%)$ & 1 & 1.54 \\
\hline & Baclofen & $2(4.8 \%)$ & $1(2.4 \%)$ & 1 & 2.05 \\
\hline & Growth hormones & $3(7.1 \%)$ & $2(4.8 \%)$ & 1 & 1.54 \\
\hline Cerebral palsy & & $7(16.7 \%)$ & $6(14.3 \%)$ & 1 & 1.2 \\
\hline Cerebral visual impairment & & $5(11.9 \%)$ & $2(4.8 \%)$ & 0.45 & 2.70 \\
\hline \multirow[t]{4}{*}{ Mental health disorder ${ }^{\mathrm{a}}$} & & $15(35.7 \%)$ & $7(16.7 \%)$ & 0.09 & 2.68 \\
\hline & Attention problems & $6(14.3 \%)$ & $3(7.1 \%)$ & 0.51 & 2.18 \\
\hline & Conduct disorders & $4(9.5 \%)$ & $1(2.4 \%)$ & 0.25 & 4.32 \\
\hline & Autism spectrum disorders & $7(16.7 \%)$ & $3(7.1 \%)$ & 0.29 & 2.60 \\
\hline \multirow[t]{5}{*}{ Disability } & Severe disability & $8(19.0 \%)$ & $5(11.9 \%)$ & 0.55 & 1.74 \\
\hline & Moderate disability & $7(16.7 \%)$ & $7(16.7 \%)$ & 1 & 1 \\
\hline & Mild disability & $10(24.8 \%)$ & $5(11.9 \%)$ & 0.12 & 2.32 \\
\hline & Multiple disabilities ${ }^{* a}$ & $17(40.5 \%)$ & $7(16.7 \%)$ & 0.04 & 3.317 \\
\hline & Disability $^{\mathrm{a}}$ & $25(59.5 \%)$ & $17(40.5 \%)$ & 0.20 & 2.038 \\
\hline \multicolumn{6}{|l|}{ Biometrics } \\
\hline 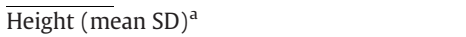 & & -1.43 & -1.08 & 0.60 & 0.780 \\
\hline Weight (mean SD) ${ }^{\mathrm{a}}$ & & -1.26 & -0.83 & 0.22 & 0.687 \\
\hline Weight for height (mean SD) & & -0.4 & -0.65 & 0.63 & \\
\hline \multicolumn{6}{|l|}{ Gastrointestinal outcome } \\
\hline$\overline{\text { Admission for bowel (sub)obstruction }}$ & & $5(11.9 \%)$ & $0(0 \%)$ & 0.06 & \\
\hline Use of laxatives/antidiarrheal drugs & & $8(19.0 \%)$ & $3(7.1 \%)$ & 0.13 & 3.06 \\
\hline Dietary restrictions & & $12(28.6 \%)$ & $5(11.9 \%)$ & 0.09 & 2.96 \\
\hline PedsQL ${ }^{\mathrm{TM}}$ Gastrointestinal Symptoms Scales ${ }^{\mathrm{a}}$ & & 6.60 & 4.52 & 0.13 & 1.808 \\
\hline Incontinence & & $6(14.3 \%)$ & $4(9.5 \%)$ & 0.72 & 1.58 \\
\hline Abdominal pain & & $8(19.0 \%)$ & $5(11.9 \%)$ & 0.42 & 1.70 \\
\hline Obstipation & & $7(16.7 \%)$ & $14(33.3 \%)$ & 0.15 & 0.40 \\
\hline Fecal urgency & & $8(19.0 \%)$ & $2(4.8 \%)$ & 0.11 & 4.71 \\
\hline Nausea & & $8(19.0 \%)$ & $6(14.3 \%)$ & 0.83 & 1.41 \\
\hline Watery stools ${ }^{\mathrm{a}}$ & & $11(26.2 \%)$ & $2(4.8 \%)$ & 0.08 & 2.564 \\
\hline \multicolumn{6}{|l|}{ Quality of life } \\
\hline \multirow[t]{5}{*}{ PedsQL ${ }^{\mathrm{TM}}$ : Quality of Life Inventory } & & 78.1 & 82.8 & 0.39 & 0.957 \\
\hline & Physical health $^{\mathrm{a}}$ & 79.5 & 89.5 & 0.23 & 0.903 \\
\hline & Emotional health & 75.8 & 74.6 & 0.78 & \\
\hline & Social health & 75.0 & 80.5 & 0.29 & \\
\hline & School functioning & 80.4 & 82.0 & 0.74 & \\
\hline
\end{tabular}

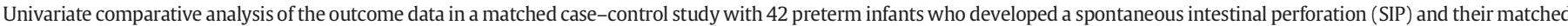
controls. For calculation of the Bayley developmental indices (BSID), 33 and 20 pairs were analyzed at 7-11 and 18-22 months, respectively, for the motor index. McNemar's and Wilcoxon tests were performed.

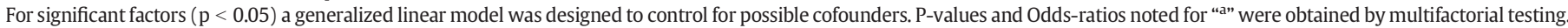
Significant data marked in bold.

PDI: psychomotor developmental index; MDI: motor developmental index.

* Significance level: $\mathrm{p}<0.05$.

0.09; GLM). Six SIP survivors and 3 controls suffered from attention problems, conduct disorders were diagnosed in 4 SIP patients and 1 control and autism spectrum disorders was noted in 7 patients from the SIP group and 3 controls. Two SIP patients presented a combined diagnosis of attention problems and autism spectrum disorders. There were no children with internalizing and externalizing problems in our cohort.

Use of medications (laxatives, antidiarrheal drugs, psychotropic or anticonvulsant drugs, muscle relaxants, asthma drugs, and growth 
Table 3

Multifactorial analysis: Odds ratios (95\% confidence intervals).

\begin{tabular}{|c|c|c|c|c|c|}
\hline \multirow[b]{2}{*}{ Outcome parameters } & \multicolumn{5}{|l|}{ Risk factors } \\
\hline & SIP & Female gender & Birth weight & Gestational age & IVH \\
\hline Normal brain ultrasound at discharge & $\begin{array}{l}0.353 \\
(0.118-1.052)\end{array}$ & $\begin{array}{l}0.812 \\
(0.187-3.537)\end{array}$ & $\begin{array}{l}0.997 \\
(0.996-1.001)\end{array}$ & $\begin{array}{l}1.200 \\
(0.803-1.793)\end{array}$ & a \\
\hline Hydrocephalus at discharge & $\begin{array}{l}8.139^{*} \\
(1.588-41.702)\end{array}$ & $\begin{array}{l}0.840 \\
(0.170-4.161)\end{array}$ & $\begin{array}{l}0.998 \\
(0.994-1.001)\end{array}$ & $\begin{array}{l}0.943 \\
(0.983-1.575)\end{array}$ & $\begin{array}{l}65.195^{* *} \\
(11.43-371.91)\end{array}$ \\
\hline Motor index (MDI) at 18-20 months & $\begin{array}{l}0.941 \\
(0.818-1.083)\end{array}$ & $\begin{array}{l}1.024 \\
(0.863-1.214)\end{array}$ & $\begin{array}{l}1.000 \\
(1.000-1.001)\end{array}$ & $\begin{array}{l}0.962 \\
(0.911-1.015)\end{array}$ & $\begin{array}{l}0.908 \\
(0.763-1.081)\end{array}$ \\
\hline Mental index (PDI) at 18-20 months & $\begin{array}{l}0.847^{*} \\
(0.723-0.992)\end{array}$ & $\begin{array}{l}1.041 \\
(0.847-1.279)\end{array}$ & $\begin{array}{l}1.000 \\
(1.000-1.001)\end{array}$ & $\begin{array}{l}0.989 \\
(0.929-1.053)\end{array}$ & $\begin{array}{l}0.948 \\
(0.746-1.176)\end{array}$ \\
\hline Physiotherapy & $\begin{array}{l}4.777^{*} \\
(1.445-15.8)\end{array}$ & $\begin{array}{l}0.671 \\
(0.146-3.088)\end{array}$ & $\begin{array}{l}0.998 \\
(0.995-1.001)\end{array}$ & $\begin{array}{l}1.456 \\
(0.917-2.312)\end{array}$ & a \\
\hline Wearing glasses & $\begin{array}{l}3.084^{*} \\
(1.161-8.193)\end{array}$ & $\begin{array}{l}1.032 \\
(0.293-3.635)\end{array}$ & $\begin{array}{l}1.000 \\
(0.998-1.002)\end{array}$ & $\begin{array}{l}1.289 \\
(0.882-1.883)\end{array}$ & $\begin{array}{l}0.474 \\
(0.134-1.685)\end{array}$ \\
\hline Regular education system & $\begin{array}{l}1.771 \\
(0.603-5.202)\end{array}$ & $\begin{array}{l}0.172 * \\
(0.035-0.841)\end{array}$ & $\begin{array}{l}0.997 \\
(0.995-1.000)\end{array}$ & $\begin{array}{l}1.211 \\
(0.803-1.825)\end{array}$ & $\begin{array}{l}8.729 * \\
(2.104-36.72)\end{array}$ \\
\hline Mental health disorder & $\begin{array}{l}2.676 \\
(0.862-8.312)\end{array}$ & $\begin{array}{l}0.210 \\
(0.036-1.225)\end{array}$ & $\begin{array}{l}1.002 \\
(0.999-1.005)\end{array}$ & $\begin{array}{l}0.987 \\
(0.652-1.494)\end{array}$ & $\begin{array}{l}1.236 \\
(0.318-4.796)\end{array}$ \\
\hline Disability & $\begin{array}{l}2.038 \\
(0.695-5.977)\end{array}$ & $\begin{array}{l}0.061^{* *} \\
(0.011-0.337)\end{array}$ & $\begin{array}{l}0.996 \\
(0.993-0.999)\end{array}$ & $\begin{array}{l}1.489 \\
(0.995-2.322)\end{array}$ & $\begin{array}{l}8.79^{*} \\
(1.722-44.86)\end{array}$ \\
\hline Multiple disabilities & $\begin{array}{l}3.317^{*} \\
(1.066-10.320)\end{array}$ & $\begin{array}{l}0.196 * \\
(0.041-0.943)\end{array}$ & $\begin{array}{l}0.997^{*} \\
(0.994-0.999)\end{array}$ & $\begin{array}{l}1.168 \\
(0.784-1.739)\end{array}$ & $\begin{array}{l}5.967^{*} \\
(1.608-22.145)\end{array}$ \\
\hline $\begin{array}{l}\text { Height } \\
\qquad(\text { mean SD) }\end{array}$ & $\begin{array}{l}0.780 \\
(0.384-1.584)\end{array}$ & $\begin{array}{l}2.727^{*} \\
(1.109-6.705)\end{array}$ & $\begin{array}{l}1.002^{*} \\
(1.000-1.004)\end{array}$ & $\begin{array}{l}0.913 \\
(0.715-1.212)\end{array}$ & $\begin{array}{l}0.466 \\
(0.192-1.129)\end{array}$ \\
\hline $\begin{array}{l}\text { Weight } \\
\text { (mean SD) }\end{array}$ & $\begin{array}{l}0.687 \\
(0.376-1.258)\end{array}$ & $\begin{array}{l}1.425 \\
(0.652-3.116)\end{array}$ & $\begin{array}{l}1.001 \\
(1.000-1.003)\end{array}$ & $\begin{array}{l}0.984 \\
(0.784-1.236)\end{array}$ & $\begin{array}{l}0.654 \\
(0.304-1.408)\end{array}$ \\
\hline PedsQL ${ }^{\mathrm{TM}}$ : Gastrointestinal Symptoms Scales & $\begin{array}{l}1.808 \\
(0.839-3.899)\end{array}$ & $\begin{array}{l}0.488 \\
(0.182-1.313)\end{array}$ & $\begin{array}{l}0.998^{*} \\
(0.996-1.000)\end{array}$ & $\begin{array}{l}1.275 \\
(0.954-1.703)\end{array}$ & $\begin{array}{l}0.815 \\
(0.312-2.125)\end{array}$ \\
\hline Watery stools & $\begin{array}{l}2.564 \\
(0.940-7.720)\end{array}$ & $\begin{array}{l}0.538 \\
(0.135-2.149)\end{array}$ & $\begin{array}{l}0.999 \\
(0.997-1.002)\end{array}$ & $\begin{array}{l}1.133 \\
(0.786-1.632)\end{array}$ & $\begin{array}{l}3.488^{*} \\
(1.071-11.362)\end{array}$ \\
\hline PedsQL ${ }^{\mathrm{TM}}$ : Quality of Life Inventory & $\begin{array}{l}0.957 \\
(0.865-1.058)\end{array}$ & $\begin{array}{l}1.050 \\
(0.923-1.195)\end{array}$ & $\begin{array}{l}1.000 \\
(1.000-1.000)\end{array}$ & $\begin{array}{l}0.993 \\
(0.956-1.031)\end{array}$ & $\begin{array}{l}0.872^{*} \\
(0.769-0.990)\end{array}$ \\
\hline PedsQL ${ }^{\mathrm{TM}}$ : Physical health score & $\begin{array}{l}0.903 \\
(0.763-1.069)\end{array}$ & $\begin{array}{l}1.043 \\
(0.840-1.296)\end{array}$ & $\begin{array}{l}1.000 \\
(1.000-1.001)\end{array}$ & $\begin{array}{l}0.986 \\
(0.929-1.051)\end{array}$ & $\begin{array}{l}0.815 \\
(0.660-1.007)\end{array}$ \\
\hline
\end{tabular}

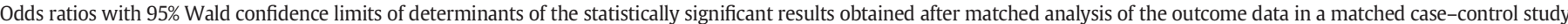

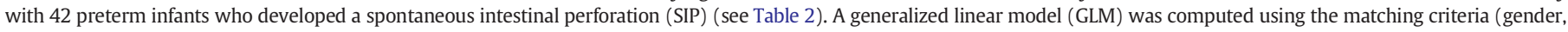

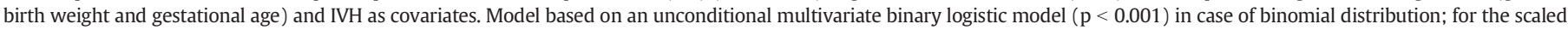
data, a gamma log model was designed.

Model effect significance levels marked in bold ${ }^{*} \mathrm{p}<0.01$; ${ }^{* *} \mathrm{p}<0.001$

IVH: Intraventricular hemorrhage.

a Data numerically unsuitable; confidence intervals tend towards infinity.

hormones) did not significantly differ between the two groups, although more SIP patients than controls used antiepileptic drugs.

\subsection{Biometrics (Tables 2, 3)}

The height and weight of SIP patients and controls were lower than those in the general population. Weight for height was also below average for age, but did not differ significantly between SIP patients and controls.

\subsection{Gastrointestinal outcome (Tables 2, 3)}

In total, $80 \%$ of SIP patients underwent a laparotomy, mostly with an ileostomy (90\%). Continuity was restored at 1-8 months after perforation. Six (15\%) SIP patients underwent neonatal revision of their stoma owing to infection or obstructive complications. Five (11.9\%) SIP patients were readmitted owing to small bowel (sub)obstruction after 5-17 years.

Table 2 summarizes the responses of parents to the gastrointestinal screening questionnaire. There were no significant differences between the two groups.

\subsection{Quality of life (Tables 2, 3)}

Parents of SIP patients and controls rated the quality of life of their children similarly at the time of evaluation $(O R=0.957 ; p=0,39$; GLM). However, multifactorial analysis revealed that patients with IVH reported a lower quality of life ( $O R=0.872 ; p=0,034 ; G L M)$.

\section{Discussion}

This retrospective study demonstrates that preterm infants who survive SIP tend to have a slightly worse neurodevelopmental outcome than matched controls. This finding seems to be related to the higher prevalence of severe IVH in the SIP group. The gastrointestinal outcome and overall quality of life did not significantly differ between the two groups.

We hypothesize that, in addition to SIP, four factors associated with the severity of illness contribute to the difference in neurodevelopmental outcome between SIP patients and controls. First, a higher percentage of SIP patients than controls suffered cerebral complications such as IVH and hydrocephalus. Severe IVH was an important comorbidity in the SIP cohort. Although IVH usually occurs in the first hours and days of life prior to intestinal perforation $[1,3,5,27]$, both conditions reflect the severity of neonatal illness. The described multifactorial model allowed us to distinguish the effects of various factors, such as SIP, IVH, and matching criteria including gestational age, birthweight, and gender, on outcome parameters such as (multiple) disability. Second, the level of inflammation is elevated in SIP patients and this can contribute to brain damage [28]. SIP is not directly correlated with a pronounced inflammatory state because most patients do not present with an elevated C-reactive protein level, but can present with marked leukocytosis [29]. However, SIP is only the start of the disease process for these patients. Surgical treatments and complications (e.g., peritonitis and septicemia) are very likely to induce delayed inflammatory reactions [30]. Third, major surgery in neonates causes developmental problems. Although the 
a

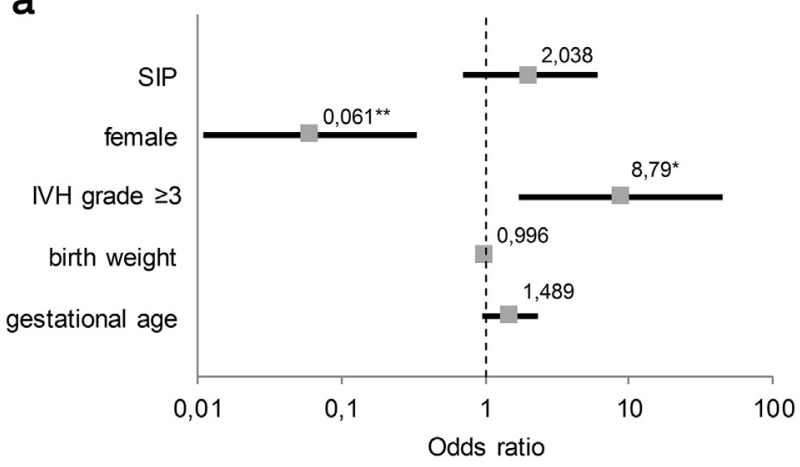

b

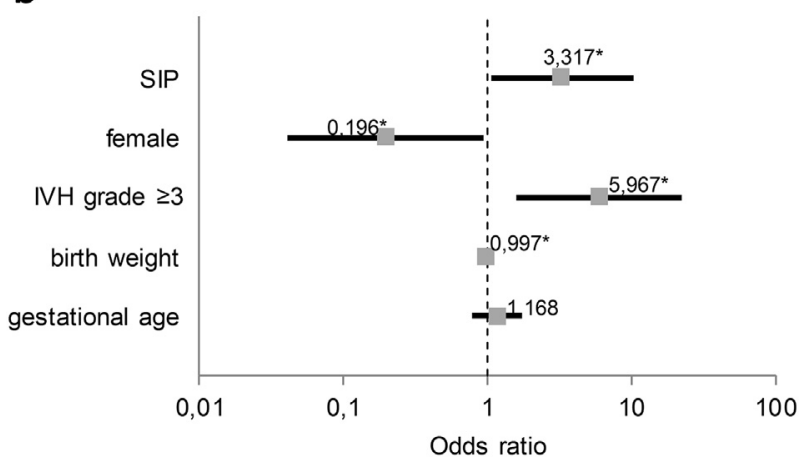

Fig. 2. Odds ratios of multivariate model. Odds ratios with 95\% Wald confidence limits of determinants of disability (a) and multiple disabilities (b) in a matched case-control study with 42 preterm infants who developed a spontaneous intestinal perforation (SIP), based on an unconditional multivariate binary logistic model $(\mathrm{p}<0.001)$. Model effect significance levels: ${ }^{*} \mathrm{p}<0.01{ }^{* *} \mathrm{p}<0.001$.

underlying mechanism is not fully understood, general anesthesia may play a role [27,31]. Finally, SIP patients suffered more complications, such as prolonged ventilation and delayed enteral feeding, than controls during their stay at the NICU, as previously reported [16].

In an American cohort of 280 SIP patients, $62.8 \%$ displayed neurodevelopmental impairment at 24 months of age [14]. This percentage is similar to that reported in the current study, although the population was younger (mean gestational age, 24.8 weeks) and had a lower mean birth weight $(710 \mathrm{~g})$ in the previous study than in the current study. The percentage of children with neurodevelopment impairment at a corrected age of 12 months was lower in a smaller study involving 18 SIP survivors (38\% had a Mental Developmental Index of $<85 \%$, and $46 \%$ had a Psychomotor Developmental Index of $<85 \%$ ) [7]. More recently, $26 \%$ of 23 SIP patients were reported to have CP at 6 years of age [15], while $15 \%$ of children had CP in both the SIP and control groups in the current study. In our study, female gender was a protective factor against disability. This effect is well-known but not fully understood [11].

A significantly higher percentage of SIP patients than controls required physiotherapy. In most cases, physiotherapy was started to stimulate gross motor development before evaluation at 9 months. Bayley developmental motor indices did not significantly differ between the two groups, although the data are probably not representative because half the patients were not formally evaluated. Our multifactorial model revealed a link between SIP and the need for physiotherapy that was independent of IVH. Factors that may minimally contribute to this early motor delay include longer hospitalization, abdominal stoma, and surgery for bowel continuity restoration. The retrospective nature of the present study hinders further analysis.

Findings regarding education did not significantly differ between the SIP and control groups. However, the percentages of children requiring specialized education in both groups were higher than normal (45\%$26 \%$ SIP-non-SIP vs $4.2 \%$ of Flemish children attend special needs schools) [32]. This is most likely because of the extreme prematurity of the SIP patients and controls [19,33]. IVH appeared to be an important risk factor for the requirement for specialized education later in life, while female gender was a protective factor in our cohort. This is likely because of the concomitant occurrence of (multiple) disability requiring adapted supportive educational measures.

In the present study, the prevalence of mental health disorders was 2-fold higher in the SIP group compared to the control group, but the difference was not significant. Extremely low birth weight infants are known to be at risk for mental health problems, with an increasing incidence proportional to the cumulative number of neonatal complications $[34,35]$. We therefore suggest that a formal psychiatric evaluation of the children should be done in order to provide more accurate information on the mental health well-being of SIP patients.

Abdominal surgery is a risk factor for intestinal obstruction owing to the formation of adhesions. The prevalence of intestinal obstruction in the SIP group (11.9\%) was slightly higher than that reported after neonatal laparotomy but was similar to that reported after surgery for NEC [36,37]. The current study revealed that development of SIP in the neonatal period had no long-term consequences on digestive wellbeing. Watery stools seemed to be a minor problem that did not affect daily life.

Overall quality of life, as reported by parents, did not significantly differ between the two groups, indicating that all of the aforementioned differences were of little significance to daily life. However, our multifactorial model revealed that patients who developed severe IVH during the neonatal period had a lower quality of life. This finding is consistent with the finding that the percentage of children with disability and multiple disabilities was higher among these patients. Our data are also consistent with a previous study concerning quality of life among children with posthemorrhagic hydrocephalus after preterm birth [38].

This study is the first to report valuable information about the longterm outcomes of SIP survivors and is the largest matched control cohort of such patients to date. The single center design of this study guarantees uniformity of treatment between the patient and matched control. The response rate was high ( $86 \%$ follow-up at 3-20 years; median, 13 years). In addition, this is the first study to investigate the gastrointestinal outcome and quality of life of SIP patients.

This study has a number of limitations. First, SIP patients were recruited over 20 years, during which treatment strategies changed. However, we limited the influence of this factor by matching SIP patients and controls in terms of the birth period. Although the response rate was high, the sample size was restricted by the decreasing frequency of SIP in our NICU. AIC of the multivariate analysis showed that model fitting was relatively good for the binary data, but also indicated that the models investigating PedsQL ${ }^{\mathrm{TM}}$ scores must be interpreted with caution. Second, the results are based on the responses of parents to a telephone questionnaire, and no medical evaluation was performed. Responders tended to be mothers. The interviewers noticed that the accuracy of the answers differed according to the education level of the parents; answers were recorded verbatim. However, the education level of mothers did not significantly differ between the two groups. The PedsQL ${ }^{\mathrm{TM}}$ Quality of Life Inventory has been validated for delivery by telephone [24]. Finally, owing to the retrospective nature of the study, some information was only available for half the patients. Consequently, BSID II scores must be interpreted with caution because data are lacking for more than half of the cohort at 18-22 months.

\section{Conclusion}

This retrospective case-matched cohort study compared the neurodevelopmental and gastrointestinal outcomes and quality of life of SIP patients and matched controls without SIP. As an important comorbidity in both groups, severe IVH led to a worse neurodevelopmental outcome requiring specialized education. Patients 
with IVH also reported a lower quality of life. Female gender was a protective factor against disability. In our cohort, SIP survivors also tended to be at risk of multiple disabilities. Gastrointestinal comorbidities did not differ between the two groups. Overall quality of life, as reported by the parents, was similar in the two groups.

Declarations of interest: none.

\section{Appendix A. English translation of a telephone survey completed by parents of SIP patients and controls}

General questions:

What is the height and weight of your child?

Has your child been admitted to hospital after discharge from the NICU?

Has your child undergone surgery after discharge from the NICU?

Does your child take daily medication?

Is your child being followed by any doctor?

Did your child receive speech therapy/physiotherapy/psychotherapy/home based care?

Does your child wear glasses/hearing aids?

Is your child on a restricted diet?

What type of school does your child attend? Does he/she receive specialized guidance at school?

What is your highest education level (mother of the patient)?

Gastrointestinal screening form (maximum score: 52, minimum score: 0 ):

In the past months, to what extent has your child been affected by the following problems?

\begin{tabular}{|c|c|c|c|c|c|}
\hline Problem & Never & $\begin{array}{l}\text { Almost } \\
\text { never }\end{array}$ & Sometimes & Often & $\begin{array}{l}\text { Almost } \\
\text { always }\end{array}$ \\
\hline \multicolumn{6}{|l|}{$\begin{array}{l}\text { Feels pain or hurt in his/her } \\
\text { stomach }\end{array}$} \\
\hline \multicolumn{6}{|l|}{ Has trouble swallowing } \\
\hline \multicolumn{6}{|l|}{$\begin{array}{l}\text { Suffers from gastroesophageal } \\
\text { reflux }\end{array}$} \\
\hline \multicolumn{6}{|l|}{$\begin{array}{l}\text { Feels the urge to vomit/does } \\
\text { vomit }\end{array}$} \\
\hline \multicolumn{6}{|l|}{ Feels his/her stomach is full of gas } \\
\hline \multicolumn{6}{|l|}{ Has hard bowel movements } \\
\hline \multicolumn{6}{|l|}{ Has watery bowel movements } \\
\hline \multicolumn{6}{|l|}{$\begin{array}{l}\text { Feels pain when he/she has a } \\
\text { bowel movement }\end{array}$} \\
\hline \multicolumn{6}{|l|}{$\begin{array}{l}\text { Has to rush to the bathroom to } \\
\text { have a bowel movement }\end{array}$} \\
\hline \multicolumn{6}{|l|}{ Is incontinent } \\
\hline \multicolumn{6}{|l|}{$\begin{array}{l}\text { Cannot eat certain foods because } \\
\text { they make him/her vomit }\end{array}$} \\
\hline \multicolumn{6}{|l|}{ Worries about stomach pain } \\
\hline Worries about bowel movements & & & & & \\
\hline Score & $\times 0$ & $\times 1$ & $\times 2$ & $\times 3$ & $\times 4$ \\
\hline
\end{tabular}

\section{Appendix B}

\begin{tabular}{ll}
\hline Model quality of multifactorial analysis & \\
\hline Parameter & AIC \\
\hline Normal ultrasound at discharge & 91 \\
Hydrocephalus & 53 \\
MDI at 1822 months & 504 \\
PDI at 1822 months & 444 \\
Physiotherapy & 88 \\
Wearing glasses & 116 \\
Mental health disorders & 97 \\
Disabilities & 102 \\
Multiple disability & 92 \\
\hline
\end{tabular}

(continued)

\begin{tabular}{lc}
\hline Model quality of multifactorial analysis & \\
\hline Parameter & AIC \\
\hline Educational system & 103 \\
Length & 311 \\
Weight & 295 \\
Peds QL ${ }^{\text {TM }}$ Gastrointestinal questionnaire & 473 \\
Watery stools & 154 \\
PedsQL ${ }^{\text {TM}}$ : Physical health score & 1179 \\
PedsQL ${ }^{\text {TM }}$ Quality of Life Inventory & 1260 \\
\hline
\end{tabular}

AIC: Akaike's information criterion.

\section{References}

[1] Holland AJA, Shun A, Martin HCO, et al. Small bowel perforation in the premature neonate: congenital or acquired? Pediatr Surg Int 2003;19:489-94.

[2] Ragouilliaux CJ, Keeney SE, Hawkins HK, et al. Maternal factors in extremely low birth weight infants who develop spontaneous intestinal perforation. Pediatrics 2007:120:1458-64.

[3] Pumberger W, Mayr M, Kohlhauser C, et al. Spontaneous localized intestinal perforation in very-low-birth-weight infants. J Am Coll Surg 2002;195:796-803.

[4] Wadhawan R, Oh W, Vohr BR, et al. Spontaneous intestinal perforation in extremely low birth weight infants: association with indometacin therapy and effects on neurodevelopmental outcomes at 18-22 months corrected age. Arch Dis Child Fetal Neonatal Ed 2013;98:127-32.

[5] Meyer CL, Payne NR, Roback SA. Spontaneous, isolated intestinal perforations in neonates with birth weight $<1,000 \mathrm{~g}$ not associated with necrotizing enterocolitis. J Pediatr Surg 1991;26:714-7.

[6] Miserez M, Barten S, Geboes K, et al. Surgical therapy and histological abnormalities in functional isolated small bowel obstruction and idiopathic gastrointestinal perforation in the very low birth weight infant. World J Surg 2003;27:350-5.

[7] Adesanya OA, O'Shea TM, Turner CS, et al. Intestinal perforation in very low birth weight infants: growth and neurodevelopment at 1 year of age. J Perinatol 2005; 25:583-9

[8] Attridge JT, Herman AC, Gurka MJ, et al. Discharge outcomes of extremely low birth weight infants with spontaneous intestinal perforations. J Perinatol 2006;26:49-54.

[9] Rees CM, Eaton S, Kiely EM, et al. Peritoneal drainage or laparotomy for neonatal bowel perforation? Ann Surg 2008;248:44-51.

[10] Merhar SL, Ramos Y, Meinzen-Derr J, et al. Brain magnetic resonance imaging in infants with surgical necrotizing enterocolitis or spontaneous intestinal perforation versus medical necrotizing enterocolitis. J Pediatr 2014;164:410-412.e1.

[11] Moore T, Hennessy EM, Myles J, et al. Neurological and developmental outcome in extremely preterm children born in England in 1995 and 2006: the EPICure studies. Obstet Gynecol Surv 2013;68:274-5

[12] Dada T, Rosenzweig JM, Al Shammary M, et al. Mouse model of intrauterine inflammation: sex-specific differences in long-term neurologic and immune sequelae. Brain Behav Immun 2014:38:142-50.

[13] Hagberg H, Gressens P, Mallard C. Inflammation during fetal and neonatal life: implications for neurologic and neuropsychiatric disease in children and adults. Ann Neurol 2012;71:444-57.

[14] Wadhawan R, Oh W, Hintz SR, et al. Neurodevelopmental outcomes of extremely low birth weight infants with spontaneous intestinal perforation or surgical necrotizing enterocolitis. J Perinatol 2014;34:64-70.

[15] Houben $\mathrm{CH}$, Feng X-N, Chan KWE, et al. Spontaneous intestinal perforation: the long-term outcome. Eur J Surg 2017;4:346-51.

[16] Rayyan M, Myatchin I, Naulaers G, et al. Risk factors for spontaneous localized intestinal perforation in the preterm infant. J Matern Fetal Neonatal Med 2018;31:2617-23.

[17] Parry G, Tucker J, Tarnow-Mordi W. CRIB II: an update of the clinical risk index for babies score. Lancet 2003;361:1789-91.

[18] Bayley N. Bayley scales of infant development. . 2nd ed.San Antonio, TX: Psychological Corporation; 1993.

[19] Larroque B, Ancel PY, Marret S, et al. Neurodevelopmental disabilities and special care of 5-year-old children born before 33 weeks of gestation (the EPIPAGE study): a longitudinal cohort study. Lancet 2008;371:813-20.

[20] Vrije Universiteit Brussel, Antropogenetica \& Katholieke Universiteit Leuven J met de steun van de VR. Vlaamse groeicurven. http://www.vub.ac.be/groeicurven/ groeicurven.html; 2004, Accessed date: 22 August 2018.

[21] Varni JW, Kay MT, Limbers CA, et al. PedsQL gastrointestinal symptoms module item development: qualitative methods. J Pediatr Gastroenterol Nutr 2012;54:664-71.

[22] Varni JW, Bendo CB, Denham J, et al. PedsQL gastrointestinal symptoms module: feasibility, reliability, and validity. J Pediatr Gastroenterol Nutr 2014;59:347-55.

[23] Varni JW, Seid M, Cheryl A. The PedsQL measurement model for the pediatric. Med Care 1999;37:126-39.

[24] Varni JW, Seid M, Kurtin PS. PedsOL ${ }^{\mathrm{TM}}$ 4.0: reliability and validity of the Pediatric Quality of Life Inventory ${ }^{\mathrm{TM}}$ version 4.0 generic core scales in healthy and patient populations. Med Care 2001;39:800-12.

[25] Pearce Neil. Analysis of matched case-control studies. BM] 2016:352.

[26] Cleophas TJ, Zwinderman AH. Machine learning in medicine - a complete overview. Zwitserland: Springer, Cham; 2015.

[27] Stolwijk LJ, Lemmers PM, Harmsen M, et al. Neurodevelopmental outcomes after neonatal surgery for major noncardiac anomalies. Pediatrics 2016;137:e20151728. 
[28] Chhor V, Schang AL, Favrais G, et al. Conséquences cérébrales à long terme de l'inflammation périnatale. Arch Pediatr 2012:19:946-52.

[29] Okuyama H, Kubota A, Oue T, et al. A comparison of the clinical presentation and outcome of focal intestinal perforation and necrotizing enterocolitis in very-lowbirth-weight neonates. Pediatr Surg Int 2002;18:704-6.

[30] Lapthorne S, Bines JE, Fouhy F, et al. Changes in the colon microbiota and intestinal cytokine gene expression following minimal intestinal surgery. World J Gastroenterol 2015;21:4150-8.

[31] Wang X, Xu Z, Miao CH. Current clinical evidence on the effect of general anesthesia on neurodevelopment in children: an updated systematic review with metaregression. PLoS One 2014;9.

[32] Vlaams onderwijs in cijfers- schooljaar 2016-2017. https://www.vlaanderen.be/ publicaties/vlaams-onderwijs-in-cijfers-2016-2017; 2017, Accessed date: 7 May 2019 .
[33] Fily A. Factors associated with neurodevelopmental outcome at 2 years after very preterm birth: the population-based Nord-Pas-de-Calais EPIPAGE cohort. Pediatrics 2006;117:357-66.

[34] Bendersky M, Lewis M. Medical and environmental risks on multiple outcomes at age three years. J Dev Behav Pediatr 1995;16:89-96.

[35] Mathewson KJ, Chow CHT, Dobson KG, et al. Mental health of extremely low birth weight survivors: a systematic review and meta-analysis. Psychol Bull 2017;143: 347-83.

[36] Wilkins B, Spitz L. Incidence of postoperative adhesion obstruction following neonatal laparotomy. Br J Surg 1986;73:762-4.

[37] Choudhry MS, Grant HW. Small bowel obstruction due to adhesions following neonatal laparotomy. Pediatr Surg Int 2006:22:729-32.

[38] Gigi M, Roth J, Eshel R, et al. Health-related quality of life after post-haemorrhagic hydrocephalus in children born preterm. Dev Med Child Neurol 2019;61:343-9. 